\title{
Alternatively activated brain-infiltrating macrophages facilitate recovery from collagenase-induced intracerebral hemorrhage
}

\author{
Hyunjung Min ${ }^{1}$, Yong Ho Jang ${ }^{1}$, Ik-Hyun $\mathrm{Cho}^{2}$, Seong-Woon $\mathrm{Yu}^{3}$ and Sung Joong Lee ${ }^{\text {* }^{*}}$
}

\begin{abstract}
Background: Intracerebral hemorrhage $(\mathrm{ICH})$ is one of the major causes of stroke. After onset of $\mathrm{ICH}$, massive infiltration of macrophages is detected in the peri-hematoma regions. Still, the function of these macrophages in ICH has not been completely elucidated.

Results: In a collagenase-induced ICH model, $\mathrm{CX} 3 \mathrm{CR} 1^{+}$macrophages accumulated in the peri-hematoma region. Characterization of these macrophages revealed expression of alternatively activated (M2) macrophage markers. In the macrophage-depleted mice, ICH-induced brain lesion volume was larger and neurological deficits were more severe compared to those of control mice, indicating a protective role of these macrophages in ICH. In the $\mathrm{ICH}$-injured brain, mannose receptor-expressing macrophages increased at a delayed time point after $\mathrm{ICH}$, indicating M2 polarization of the brain-infiltrating macrophages in the brain microenvironment. To explore this possibility, bone marrow-derived macrophages (BMDM) were co-cultured with mouse brain glial cells and then tested for activation phenotype. Upon co-culture with glia, the number of mannose receptor-positive M2 macrophages was significantly increased. Furthermore, treatment with glia-conditioned media increased the number of BMDM of M2 phenotype.
\end{abstract}

Conclusions: In this study, our data suggest that brain-infiltrating macrophages after ICH are polarized to the M2 phenotype by brain glial cells and thereby contribute to recovery from ICH injury.

Keywords: Immune response, Macrophages, Wound healing

\section{Background}

Intracerebral hemorrhage $(\mathrm{ICH})$ is a type of stroke characterized by blood leakage into the brain parenchyma due to blood vessel rupture within the brain. Although it accounts for only about 15 to $20 \%$ of all strokes, ICH has a higher mortality and disability rate than other types of stroke. In $\mathrm{ICH}$, the initial insult due to the mechanical force of the expanding hematoma and plasma proteins is usually followed by secondary

\footnotetext{
* Correspondence: sjlee87@snu.ac.kr

'Department of Neuroscience and Dental Research Institute, School of Dentistry, Seoul National University, 1 Gwanak-ro, Gwanak-gu, Seoul 08826, Republic of Korea

Full list of author information is available at the end of the article
}

damage, which involves inflammatory responses in the peri-hematoma region $[1,2]$. The inflammatory responses accompany brain-resident astrocyte activation, compromise of the blood brain barrier (BBB) in the perihematoma region, and recruitment of peripheral immune cells such as neutrophils and macrophages to the injury site [3-7]. We have previously reported that $\mathrm{ICH}-$ induced neutrophil infiltration is partly responsible for secondary brain damage after ICH [8]. Also, we have shown that toll-like receptor 2 (TLR2)-mediated MMP9 activation in astrocytes is involved in the BBB compromise and subsequent neutrophil infiltration [8]. Other literature has documented that brain-infiltrated neutrophils exert neurotoxic effects by producing reactive oxygen species and pro-inflammatory mediators $[9,10]$. 
Although a detrimental role of brain-infiltrating neutrophils after $\mathrm{ICH}$ has been well-documented, the role of brain-infiltrating macrophages has not been completely elucidated.

It is well known that macrophages display plasticity in their characteristics and can change phenotype and functions depending on the microenvironment $[11,12]$. Typically, stimulation with lipopolysaccharide (LPS) and interferon- $\gamma$ activates macrophages to express proinflammatory mediators such as TNF- $\alpha$ and iNOS, referred to as "classically activated" M1 macrophages [13]. Conversely, interleukin (IL)-4 and IL-10 stimulation induce "alternatively activated" M2 macrophages that promote tissue healing and angiogenesis [14]. These two types of macrophage activations are detected in various neurological injury models. In spinal cord injury, prolonged activation of M1 macrophages due to TNF- $\alpha$ and iron impedes recovery after injury [15], while TLR2dependent M2 polarization of microglia protects against spinal cord injury [16]. In a murine ischemic stroke model, macrophages assume the M2 phenotype in early stages of ischemic stroke but gradually transform into the M1 phenotype in the peri-infarct region, which exacerbates neuronal death [17]. The phenotype of macrophages in hemorrhagic stroke is not yet completely understood. In this study, we used a mouse model of collagenase-induced $\mathrm{ICH}$ to explore the function and phenotype of braininfiltrating macrophages after hemorrhagic stroke.

\section{Methods}

\section{Animals}

C $x 3 c r 1^{+/ G F P}$ mice with GFP knocked into the gene encoding CX3CR1 were from Jackson Laboratory. Seven- to 11-week-old C57BL/6 mice were purchased from Daehan Bio Link (Eumsung, Korea). All mice were housed at $23 \pm 2{ }^{\circ} \mathrm{C}$ with a $12 \mathrm{~h}$ light-dark cycle and fed food and water ad libitum. All surgical and experimental procedures were reviewed and approved by the Institutional Animal Care and Use Committee at Seoul National University.

\section{ICH model}

The procedure for inducing $\mathrm{ICH}$ through collagenase injection in mice was adapted from a protocol previously described [10]. Briefly, WT and $C x 3 c r 1^{+/ G F P}$ mice (8-12week-old males, 22-25 g) were anesthetized using Avertin (250 mg/kg body weight, i.p.) and placed on a stereotaxic apparatus (myNeuroLab, St. Louis, MO, USA). Animals were injected with PBS or collagenase VII-S (0.075 U in 0.5 $\mu \mathrm{l} \mathrm{PBS;} \mathrm{Sigma,} \mathrm{St.} \mathrm{Louis,} \mathrm{MO,}$ USA) at a rate of $0.2 \mu \mathrm{l} / \mathrm{min}$ into the right caudate putamen (stereotaxic coordinates in millimeters with reference to bregma: $\mathrm{AP},-0.9 ; \mathrm{ML},+2.8 ; \mathrm{DV},-4.0)$ using a 26-G needle. After $5 \mathrm{~min}$, the needle was removed in three intermediate steps for 3 min to minimize backflow. The incision was disinfected with povidone iodide solution and sutured, and the animals were kept on a warm pad during recovery.

\section{Injury volume analysis}

To prepare brain tissue sections, animals were deeply anesthetized with urethane and perfused intra-cardially with saline followed by cold $4 \%$ paraformaldehyde (PFA) in a $0.1 \mathrm{M}$ phosphate buffer. The brains were removed, post-fixed overnight in the same fixative at $4{ }^{\circ} \mathrm{C}$, rinsed twice with PBS, and placed serially in 10 , 20, and $30 \%$ sucrose in PBS for $72 \mathrm{~h}$ at $4{ }^{\circ} \mathrm{C}$. The brains were then quickly frozen and cut into serial coronal sections $(50 \mu \mathrm{m}$ thickness $)$ in a cryostat (CM3050S, Leica, Germany). Sections were collected as free-floating sections in cold PBS and then used for histological analysis. Five coronal brain slices from different levels of the injured hemorrhagic area were selected from each mouse brain and used for cresyl violet staining. The injury area was quantified using Image J software (National Institutes of Health, Bethesda, MD, USA), and the injury volume was calculated in cubic millimeters $\left(\mathrm{mm}^{3}\right)$ by multiplying section thickness by measured injury area.

\section{Behavioral test}

The adhesive removal test (ART) was performed as previously described [18]. Mice were habituated for at least 30 min prior to applying the sticky tape on the sole of the left fore paw. The removal time was defined as the period from the mouse's initial reaction to the presence of the adhesive tape strips to the time it detached the tape using its mouth or forelimb. The duration of the procedure to test one mouse was $300 \mathrm{~s}$ maximum. ART was performed at 1 and 3 days after $\mathrm{ICH}$ or sham operation. Neurological function was measured by scoring focal deficits on a 28-point neurological scoring system [19]. Habituated mice were scored on the following measures: (1) Body symmetry, (2) Gait, (3) Climbing, (4) Circling behavior, (5) Front limb symmetry, (6) Compulsory circling, and (7) Whisker response. One and 3 days after $\mathrm{ICH}$, scoring was performed on each subject on a scale from 0 to 4 , and the scores were averaged.

\section{Peripheral macrophage/monocyte depletion}

Peripheral monocytes/macrophages were systemically depleted using a clodronate liposome suspension (ClodronateLiposome.com, Amsterdam, Netherlands). Mice were injected intra-peritoneally with $1 \mathrm{mg}$ of clodronate liposome $\left(\mathrm{Cl}_{2} \mathrm{MDP}\right)$ or saline 1 day before $\mathrm{ICH}$ surgery. One day after $\mathrm{ICH}$, an additional injection was administered in order to increase the depletion rate. To measure depletion efficiency, the brains 
and blood samples were collected and monocyte/ macrophage populations were evaluated by flow cytometry or anti-CD68 immunofluorescence staining.

\section{Immunohistochemistry}

Immunostaining was carried out using previously established protocols [20]. The sections were incubated in a blocking solution (5\% normal donkey serum, $2 \%$ BSA, and $0.1 \%$ Triton X-100) for $1 \mathrm{~h}$ at room temperature (RT). The sections were then incubated overnight at $4{ }^{\circ} \mathrm{C}$ with goat anti-Arginase-1 (1:50; Santa Cruz Biotechnology, CA, USA) and rat anti-CD68 (1:100; AbD Serotec, Oxford, UK). The sections were incubated for $1 \mathrm{~h}$ at RT with Cy3conjugated secondary antibodies (1:200; Jackson ImmunoResearch, West Grove, PA, USA) and then mounted on gelatin-coated slides and cover-slipped with VectaShield medium (Vector Labs, Burlingame, CA, USA). All images were acquired using confocal laser scanning microscopy (LSM700; Carl Zeiss, Germany).

\section{Quantitative real-time RT-PCR}

The cDNA was synthesized using total RNA from mouse brain tissue or in vitro cultured cells. The reverse transcription mixture consisted of 1-3 $\mu \mathrm{g}$ of total RNA, oligo-dT, M-MLV, RNAse inhibitor, DTT, and RT buffer and was synthesized at $37^{\circ} \mathrm{C}$ for $1 \mathrm{~h}$. Real-time RT-PCR was performed using SYBR Green PCR Master Mix (ABI, Waltham, MA, USA) as previously described [20]. Reactions were performed in duplicate in a total volume of $12 \mu \mathrm{l}$ containing $10 \mathrm{pM}$ primer, $5 \mu \mathrm{l} \mathrm{cDNA}$, and $6 \mu \mathrm{l}$ SYBR Green PCR Master Mix (ABI). The mRNA level of each target gene was normalized to that of GAPDH mRNA. Fold-induction was calculated using the $2^{-\Delta \Delta C T}$ method, as previously described [21]. All real-time RT-PCR experiments were performed at least three times and are presented as mean \pm SEM unless otherwise noted. The following sequences of primers were used for real-time RT-PCR: Arginase- 1 forward: $5^{\prime}-\mathrm{G}$ GG CTC TGA TGA GAA GGA GA-3'; Arginase-1 reverse: 5'-GTA GAT GCC ACG CTG GTA CA-3'; Ym1 forward: 5'-GAA GGA GCC ACT GAG GTC TG-3'; Ym1 reverse: 5'-CAC GGC ACC TCC TAA ATT GT-3'; iNOS forward: 5'-GGC AAA CCC AAG GTC TAC GTT-3'; iNOS reverse: $5^{\prime}$-TCG CTC AAG TTC AGC TTG GT-3'; TNF- $\alpha$ forward: 5'-AGC AAA CCA CCA AGT GGA GGA-3'; TNF- $\alpha$ reverse: 5'-GCT GGC ACC ACT AGT TGG TTG T-3'; CD16 forward: 5' - TTT GGA CAC CCA GAT GTT TCA G-3'; CD16 reverse: 5' GTC TTC CTT GAG CAC CTG GAT C-3'; CD86 forward: 5' - TTG TGT GTG TTC TGG AAA CGG AG-3' and CD86 reverse: 5' - AAC TTA GAG GCT GTG TTG CTG GG-3'.

\section{Primary mouse brain glial cell culture}

Primary mouse brain glial cultures were prepared as previously described [22]. Briefly, brains were prepared from postnatal day 1-3 wild-type mice. After removing the meninges from the cerebral hemisphere, tissue was dissociated into a single-cell suspension through gentle repetitive pipetting. Cells were cultured in Dulbecco's Modified Eagle's medium (DMEM) and supplemented with $10 \mathrm{mM}$ HEPES, $10 \%$ FBS, 2 mM L-glutamine, $1 \mathrm{X}$ non-essential amino acids (NEAA), and 1X antibiotic/antimycotic in $75 \mathrm{~cm}^{2}$ flasks at $37{ }^{\circ} \mathrm{C}$ in a $5 \% \mathrm{CO}_{2}$ incubator. The medium was changed every 5 days.

\section{BMDM culture}

The protocols for BMDM culture and cryopreservation were adapted from a previous study [23]. Briefly, femurs and tibias were obtained from 8 to 12-weekold C57BL/6 or $\mathrm{C} x 3 \mathrm{crl}^{+/ G F P}$ mice. The bones were flushed with a syringe filled with DMEM to extrude bone marrow into a $50 \mathrm{ml}$ sterile tube. The cells were gently homogenized using a plastic pipette and aliquoted in freezing media containing $90 \% \mathrm{FBS}$ and $10 \%$ DMSO. The aliquots were maintained at $-80{ }^{\circ} \mathrm{C}$ for $24 \mathrm{~h}$ and then transferred to a liquid nitrogen tank. Bone marrow cells were thawed, washed with warm-DMEM, and then cultured in DMEM supplemented with $10 \mathrm{mM}$ HEPES, $10 \%$ FBS, 2 mM L-glutamine, $1 \mathrm{X}$ NEAA, $1 \mathrm{X}$ antibiotic/antimycotic, and $30 \mathrm{ng} / \mathrm{ml}$ recombinant human macrophage colony stimulating factor (rhM-CSF; Peprotech, Rocky Hill, NJ, USA) at $37{ }^{\circ} \mathrm{C}$ in a $5 \% \mathrm{CO}_{2}$ atmosphere for 5 days. For mRNA quantification or flow cytometry analysis, BMDMs were seeded in a 6-well plate $\left(2.5-5 \times 10^{5} /\right.$ well) with growth media supplemented with $10 \mathrm{ng} / \mathrm{ml}$ rhM-CSF for 2 days.

\section{Co-culture experiment}

While the BMDM were removed from the plate with a scraper, the primary mixed glial cells were simultaneously detached using trypsin. Cells were counted using a hemocytometer $\left(2.5-5 \times 10^{5}\right.$ cells $)$ and then added to a $60 \mathrm{~mm}$ culture dish at a 1:1 ratio in BMDM growth medium-containing $10 \mathrm{ng} / \mathrm{ml} \mathrm{rhM}$ CSF. Co-cultures were incubated at $37{ }^{\circ} \mathrm{C}$ in a $\mathrm{CO}_{2}$ incubator.

\section{Glial cell-conditioned media treatment}

Two weeks after glial culture, conditioned media were collected. To prevent cell contamination, conditioned media were centrifuged for a short time and then stored in a $-80{ }^{\circ} \mathrm{C}$ deep freezer. A 1:1 mixture of glia-conditioned media and BMDM growth media was used for treatment. 


\section{Flow cytometry}

The mice were deeply anesthetized with urethane and intra-cardially perfused with ice-cold saline. Ipsilateral hemispheres were trimmed on the anterior and posterior sides from the needle hole, and then residues of brain hemispheres were homogenized mechanically to a single cell-suspension. In addition, blood samples were collected by retrobulbar puncture before mice were perfused with saline and red blood cells were removed by $\mathrm{RBC}$ lysis buffer $\left(150 \mathrm{mM} \mathrm{NH}{ }_{4} \mathrm{Cl}, 10 \mathrm{mM} \mathrm{KHCO}_{3}\right.$, $100 \mu \mathrm{M}$ EDTA). For the analysis of cultured cells, cocultures or BMDMs were removed from the plates with cold PBS and re-suspended using a pipette. Cells were washed with ice-cold $2 \%$ FBS in PBS and incubated with Fc Blocker ${ }^{\mathrm{TM}}$ (BD Bioscience, San Jose, CA) for $10 \mathrm{~min}$ at $4{ }^{\circ} \mathrm{C}$ prior to staining with CD11b-FITC, CD45-PE, CD206-APC, F4/80-PE/Cy7 (Biolegend Inc., San Diego, CA). BD FACSCalibur flow cytometer (BD Bioscience) was used to measure the microglia as $\mathrm{CD} 11 \mathrm{~b}^{+} / \mathrm{CD} 45^{\text {low }}$, peripheral macrophage as $\mathrm{CD} 11 \mathrm{~b}^{+} / \mathrm{CD} 45^{\text {high }}$, and $\mathrm{BMDM}$ as $\mathrm{CD} 45^{+} / \mathrm{GFP}^{+}$or $\mathrm{CD} 11 \mathrm{~b}^{+} / \mathrm{CD} 45^{+}$. Data were acquired and analyzed with BD CellQuest ${ }^{\mathrm{TM}}$ system (BD Biosciences).

\section{Statistical analysis}

Statistically significant differences between two groups were determined using two-tailed Student's $t$-test. Differences among multiple groups were identified using oneway ANOVA followed by Bonferroni correction. All data are presented as mean \pm SEM, and differences were considered significant when the $p$-value was less than 0.05 .

\section{Results}

\section{ICH injury induces macrophage infiltration into injured} brain parenchyma

To detect macrophages infiltrating brain parenchyma after ICH injury, we utilized $C \times 3 \mathrm{crl}^{+/ G F P}$ mice in which CX3CR1-expressing macrophages and microglia can be monitored using GFP fluorescence. Following $\mathrm{ICH}$, the cells with GFP fluorescence increased as soon as 1 day post-injury (dpi) in the surrounding area of hematoma, indicating upregulation of CX3CR1 in microglia/macrophages in this area (Fig. $1 \mathrm{~b}$ and c). Three days after ICH, CX3CR $1^{+}$cells greatly increased in the injured brain area. In particular, round CX3CR $1^{+}$cells, morphologically presumed to be brain-infiltrating activated macrophages, accumulated in the peri-hematoma region, which was further increased on $7 \mathrm{dpi}$ (Fig. 1d and e). To differentiate macrophages from microglia in this CX3CR $1^{+}$population, we analyzed the $\mathrm{F} 4 / 80$-positive monocytic cell population in the injured brain using flow cytometry based on the expression level of CD45 (Fig. 1f) [24]. The percentage of $\mathrm{CD} 45^{\text {high }} / \mathrm{F} 4 / 80^{+}$leukocytes representing the macrophage population increased to
$11.5 \%$ on $1 \mathrm{dpi}$ and $31 \%$ on 3 dpi from $1.8 \%$ (sham). In the same time frame, the number of $\mathrm{CD} 45^{\text {low }} / \mathrm{F} 4 / 80^{+}$ cells representing the microglia population was not as significantly altered; it increased only to $58 \%$ on $3 \mathrm{dpi}$ from $40 \%$ (sham). These data demonstrate that the increase in $\mathrm{CX} 3 \mathrm{CR} 1^{+}$cells after $\mathrm{ICH}$ is mainly due to macrophage infiltration rather than microglia proliferation.

\section{Brain-infiltrating macrophages following $\mathrm{ICH}$ show M2 phenotype}

To characterize the phenotype of the brain-infiltrating macrophages, we analyzed expression of typical M1 (CD16, CD86, and iNOS) and M2 (Arginase-1 and Ym1) markers in the injured brain. Upon initiation of $\mathrm{ICH}$, the mRNA expression of Arginase-1 increased more than 300-fold in the injured brain at $7 \mathrm{dpi}$. Likewise, Ym1, another M2 marker, expression was increased 15-fold at 3 dpi (Fig. 2a and b). For M1 markers, only CD16 expression was increased 4.7-fold at 3 dpi (Fig. 2c), whereas other M1 markers such as CD86 and iNOS expressions were not significantly altered (Fig. $2 \mathrm{~d}$ and e). Arginase-1 expression was mainly detected in $\mathrm{CX}_{3} \mathrm{CR}^{+}{ }^{+}$macrophages/microglia in the peri-hematoma region (Fig. 2f, arrows), suggesting that macrophages/microglia in the injured brain are polarized to the M2 phenotype. To confirm these data, we analyzed the expression of mannose receptor (CD206), another M2 marker, in the $\mathrm{CD}_{11} \mathrm{~b}^{+}$monocyte cell population in the ipsilateral $\mathrm{ICH}-$ injured brain using flow cytometry (Fig. $2 \mathrm{~g}$ ). The percentages of mannose receptor-expressing macrophages $\left(\mathrm{CD} 206^{+} / \mathrm{CD} 11 \mathrm{~b}^{+} / \mathrm{CD} 45^{\text {high }}\right)$ increased to $68.5 \%$ at 7 days post- $\mathrm{ICH}$, whereas the $\mathrm{CD}^{2} 06^{+}$microglia population $\left(\mathrm{CD} 206^{+} / \mathrm{CD} 11 \mathrm{~b}^{+} / \mathrm{CD} 45^{\text {low }}\right)$ increased to only $16.7 \%$. Taken together, these data suggest that the majority of macrophages in the $\mathrm{ICH}$-injured brain are polarized to the M2 phenotype at delayed time points ( 3 and $7 \mathrm{dpi}$ ).

\section{Macrophage depletion attenuates recovery after ICH}

To assess the role of these M2 macrophages, we depleted the monocyte cell population by treating with clodronate liposome $\left(\mathrm{Cl}_{2} \mathrm{MDP}\right)$ and then subjected mice to $\mathrm{ICH}$ injury. Upon $\mathrm{Cl}_{2} \mathrm{MDP}$ injection (i.p.), $97 \%$ of blood monocytes were depleted from the blood (Fig. 3b). In the ICH-injured brain, the percentage of $\mathrm{CD} 11 \mathrm{~b}^{+} / \mathrm{CD} 45^{\text {high }}$ macrophages was increased to $8.1 \%$ (saline- $\mathrm{ICH}$ ) from $1.5 \%$ (salinesham) (Fig. 3c). However, in the $\mathrm{Cl}_{2} \mathrm{MDP}$-treated mice brain, the macrophage population only increased to $1.6 \%$ $\left(\mathrm{Cl}_{2} \mathrm{MDP}-\mathrm{ICH}\right)$ from $0.9 \%\left(\mathrm{Cl}_{2} \mathrm{MDP}\right.$-sham $)$. Meanwhile, the microglia $\left(\mathrm{CD} 11 \mathrm{~b}^{+} / \mathrm{CD} 45^{\text {low }}\right)$ populations were not significantly altered by $\mathrm{Cl}_{2} \mathrm{MDP}$ treatment (Fig. 3c). Decreased macrophage infiltration in the $\mathrm{Cl}_{2} \mathrm{MDP}$ injected mouse brain was also confirmed by 


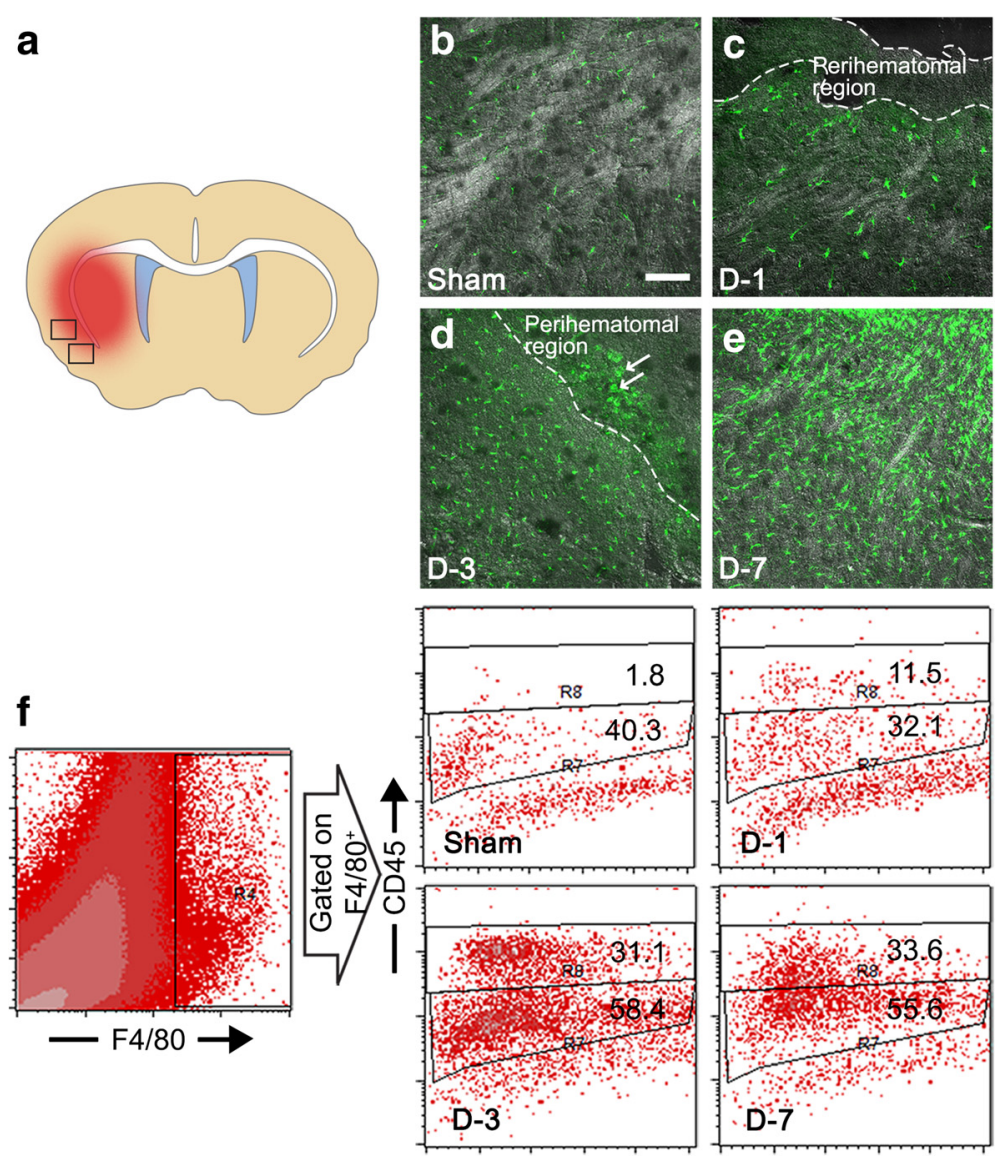

Fig. $1 \mathrm{CX} 3 \mathrm{CR} 1^{+}$cells increase in the peri-hematoma region after $\mathrm{ICH}$. a $\mathrm{CX} 3 \mathrm{CR} 1^{+}$cells were detected in peri-hematoma regions of hemorrhagic brain sections. b-e Representative images on brain sections were obtained from $\mathrm{C} \times 3 \mathrm{Cr}]^{+/ G F P}$ mice at 1, 3, and 7 days after ICH or sham operation. Three days after $\mathrm{ICH}$, amoeboid cells were detected in the peri-hematoma region (arrows) (Scale bar, $100 \mu \mathrm{m})$. $\mathbf{f}$ Myeloid cells ( $\left(\mathrm{F} 4 / 80^{+}\right.$) in the lesion were analyzed and further divided into CD45-low microglia and CD45-high macrophage populations according to the expression level of CD45 according to flow cytometry. The flow cytometry data are representative of three independent experiments

immunohistochemistry (Fig. 3d). The $\mathrm{CD}^{+} 8^{+}$macrophages in the $\mathrm{ICH}$-injured brain were significantly reduced in $\mathrm{Cl}_{2} \mathrm{MDP}$-treated mice (Fig. 3d). Cresyl violet staining of ipsilateral brain sections from $\mathrm{Cl}_{2} \mathrm{MDP}$ - or saline-treated mice showed that monocyte-depleted mice had larger infarct volumes after ICH compared to control mice (Fig. 3e and f). Consistent with the histological data, the $\mathrm{Cl}_{2} \mathrm{MDP}$-treated mice displayed more severe motor function impairment as measured by the adhesive removal test (Fig. 3g) and focal deficits scoring (Fig. 3h) at $3 \mathrm{dpi}$. These findings indicate that brain-infiltrating M2 macrophages play a protective role by presumably facilitating recovery from $\mathrm{ICH}$ injury.

\section{Macrophages are polarized to M2 phenotype by soluble factors secreted by glial cells}

Since the macrophages in the injured brain were M2 phenotype, it is conceivable that brain microenvironment polarizes brain-infiltrating macrophages to the M2 phenotype. Supporting this possibility, a previous study has shown that bone marrow-derived macrophages (BMDM) exhibit an M2-like phenotype after co-culture with an astroglioma cell line [25]. To test if glial cells affect macrophage polarization toward the M2 phenotype, BMDM from $C x 3 c r 1^{+/ G F P}$ mice were co-cultured with primary cultured mouse brain glial cells, and mannose receptor expression was measured using flow cytometry. After co-culture with primary glia for 3 days, the percentage of $\mathrm{CD}_{206}{ }^{+}$cells in the BMDM population $\left(\mathrm{GFP}^{+} / \mathrm{CD} 45^{+}\right)$increased to $20 \%$, indicating polarization to the M2 phenotype (Fig. $4 \mathrm{a}$ and b). Notably, the percentage of $\mathrm{CD} 206^{+} \mathrm{BMDM}$ also increased to more than $60 \%$ upon incubation in glia-conditioned media for 3 days (Fig. 4c), indicating that direct cell contact between BMDM and glia is not required for M2 polarization. In addition, the expression of other M2 genes Arginase-1 and Ym1 increased by 1.5- and 6-fold, respectively (Fig. 4d and e). However, expression of iNOS and TNF- $\alpha$ mRNA, M1 marker genes, was not 

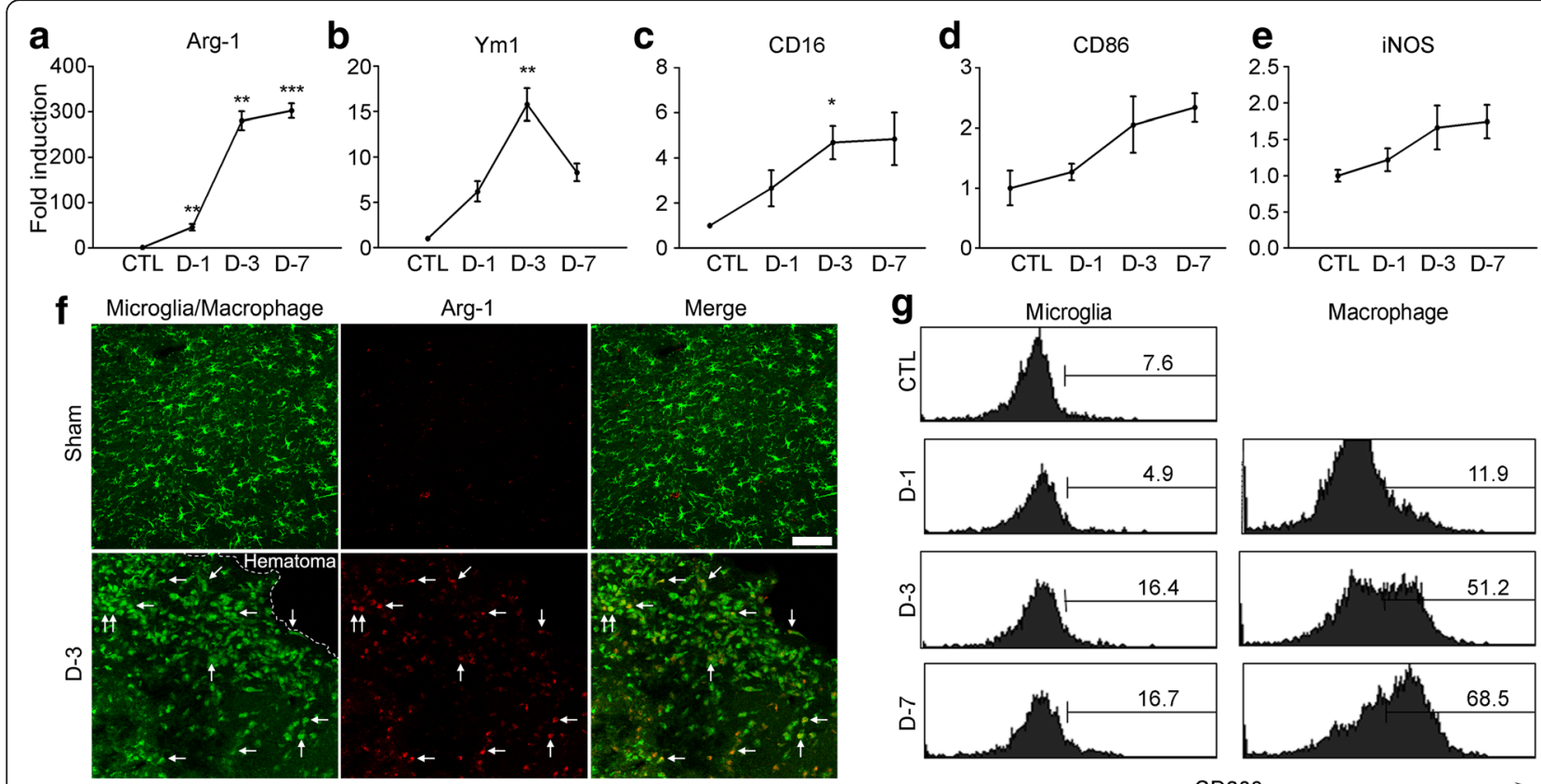

Macrophage
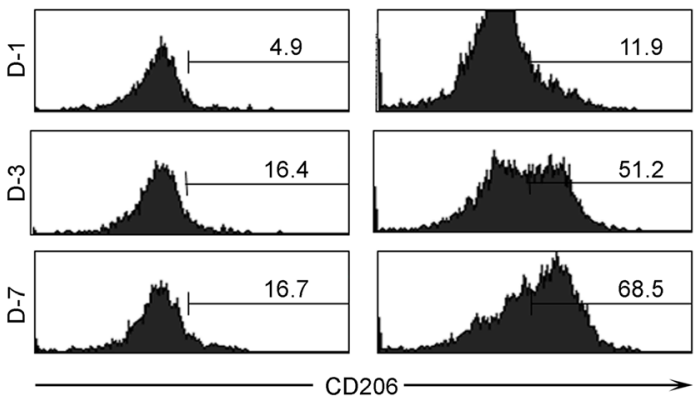

Fig. $2 \mathrm{M} 2$ macrophages are increased in the ipsilateral brain after $\mathrm{ICH}$. a-e mRNA expressions of M2 markers (Arg-1 and Ym1) or M1 markers (CD16, CD86 and iNOS) in the ICH mice brains were measured using real-time RT-PCR. Total RNA was isolated from ipsilateral hemorrhagic tissue and used for quantitative real-time RT-PCR ( $n=3$ per group, $\left.{ }^{* *} p<0.01,{ }^{* * *} p<0.001\right)$. f Representative images of Arg-1 immunofluorescence (red) in the $C X 3 C R 1^{+/ G F P}$ mice brain sections obtained from ICH or sham-control mice. Arg-1 immunofluorescence merged with myeloid cell-specific GFP signals is denoted (arrows) (Scale bar, $100 \mu \mathrm{m}$ ). g Dissociated cells from the injured tissue were obtained 1, 3, and 7 days after ICH injury and were used for flow cytometry with anti-CD11b-FITC, -CD45-PE, and -CD206-APC antibodies. Representative flow cytometry histograms show $\mathrm{CD}_{20} 6^{+}$cells gated on CD11 $\mathrm{b}^{+} / \mathrm{CD} 45^{+}$populations. The flow cytometry data are representative of three independent experiments

significantly upregulated after glia-conditioned media treatment (Fig. 4f and g). These data suggest that gliaderived soluble factor(s) induce M2 polarization of the BMDM, which might account for the M2 polarization of macrophages observed in the $\mathrm{ICH}$-injured brain.

\section{Discussion}

Although the function of brain-infiltrating immune cells in ischemic stroke has been well documented, their role in hemorrhagic stroke is not completely understood. To our knowledge, this study is the first to assert that macrophages infiltrating brain parenchyma after ICH might play a beneficial role in recovery from $\mathrm{ICH}$ injury. Our data are in contrast to the recent report by Hammond et al., which showed that brain-recruited inflammatory monocytes exacerbate disability following $\mathrm{ICH}$ [26]. In this study, they showed that immune cells recruited to the injured brain after ICH are mainly $\mathrm{CCR} 2^{+} / \mathrm{Ly} 6 \mathrm{C}^{+}$inflammatory macrophages that peaked at 1-3 dpi. Such discrepancy might simply be due to differences in their injury model, since they characterized the phenotype of macrophages in an ICH model using autologous blood infusion. They also showed that these inflammatory macrophages contribute to acute neurological deficit by depleting inflammatory macrophages using CCR2 antibody. CCR2 is expressed on inflammatory macrophages but not on $\mathrm{CX} 3 \mathrm{CR} 1^{+}$monocytes [27]. Thus, the treatment of this antibody might have depleted only inflammatory monocytes but not CCR2-negtive M2 macrophages. Therefore, it is possible that the contribution of anti-inflammatory or wound-healing M2 macrophages that are recruited after 3 dpi might have been overlooked in their study.

It should also be noted that clodronate liposome may deplete both monocytes and other myeloid cells, such as dendritic cells. A recent study showed that dendritic cells were also recruited to the injured brain after $\mathrm{ICH}$ [28]. Thus, we cannot rule out the possibility that the effects of clodronate liposome on ICH injury could be due to both monocyte and dendritic cell depletion. In the present study, we did not analyze the relative contributions of brain-infiltrating macrophages and dendritic cells, which warrants future investigation.

Our data clearly show that brain-infiltrating macrophages play distinct roles depending on the stroke model used. In a murine ischemic stroke model, macrophages played a detrimental role by exacerbating neuronal cell death [17]. In this model, macrophages in the periinfarct brain region were gradually transformed into M1 phenotype, which contributed to the detrimental effects 
a
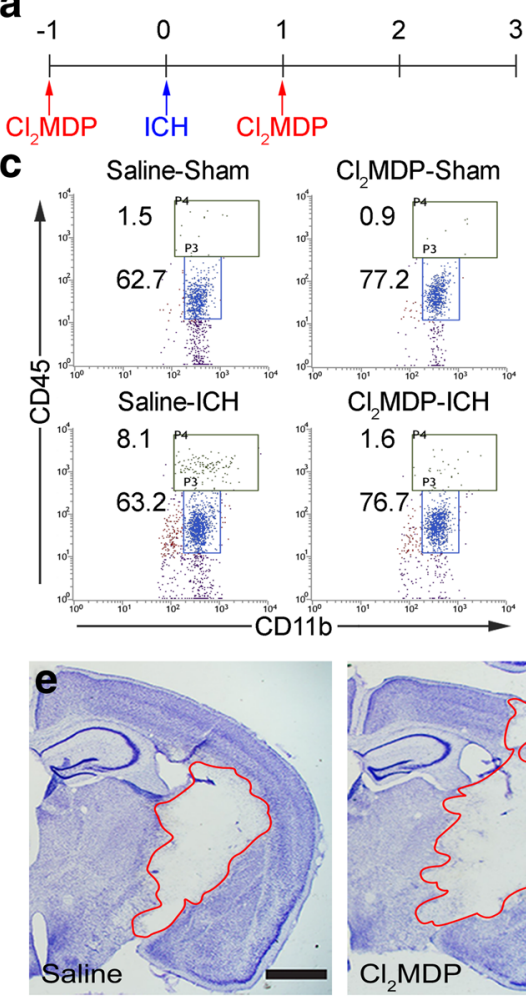

g

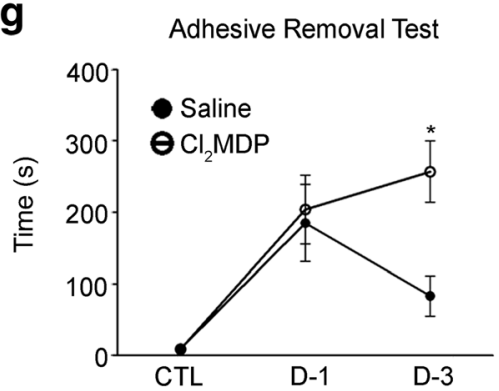

b
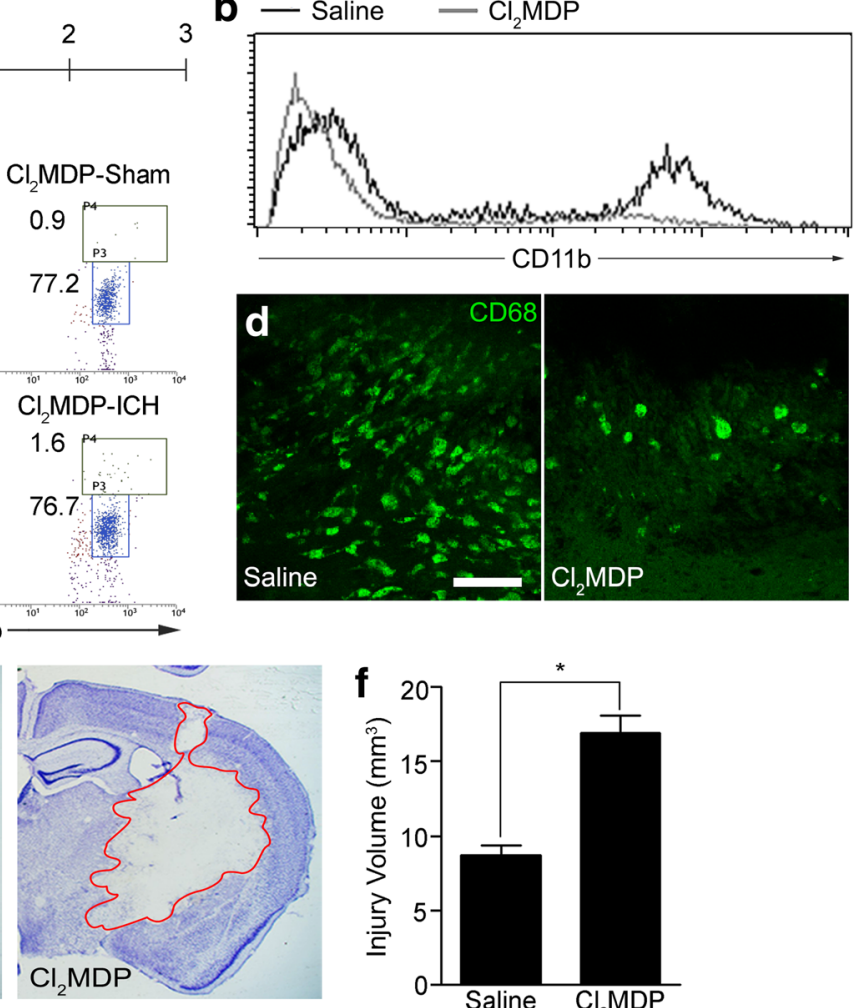

h

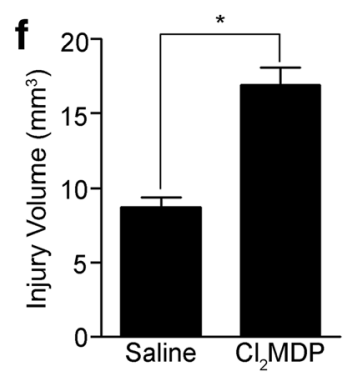

Focal Deficit

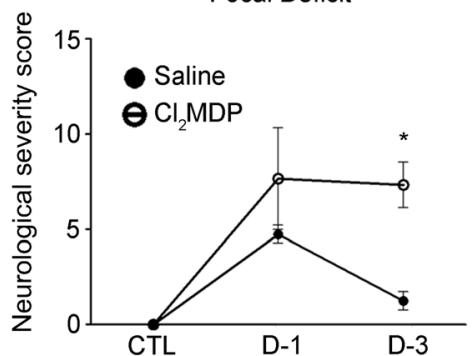

Fig. 3 Depletion of peripheral monocytes in ICH injury. a Schematic diagram of the myeloid cell depletion protocols using $\mathrm{Cl}_{2} \mathrm{MDP}$. $\mathbf{b}$ Blood cells obtained from saline (black line)- or $\mathrm{Cl}_{2} \mathrm{MDP}$-treated ICH mice (gray line) were stained with anti-CD11b-FITC antibody and analyzed with flow cytometry. c Cell suspensions obtained from saline- or $\mathrm{Cl}_{2} \mathrm{MDP}$-treated mouse brain were stained with anti-CD11b-FITC and anti-CD45-PE antibody and analyzed with flow cytometry. Representative flow cytometry dot plots are shown. $\mathbf{d}$ ICH injured brain sections were collected and stained with anti-CD68 antibody. Representative images of three independent experiments are shown (Scale bar, $50 \mu \mathrm{m}$ ). e One day after $\mathrm{Cl}_{2} \mathrm{MDP}$ injection, mice were subjected to $\mathrm{ICH}$, and the brains were sectioned and stained with cresyl violet at 3 dpi. Representative pictures are shown (Scale bar, $1 \mathrm{~mm}$ ). $\mathbf{f}$ Hemorrhagic injured volumes were quantified and presented in a graph $\left(n=4\right.$ per group, $\left.{ }^{*} p<0.05\right)$. $\mathbf{g}, \mathbf{h ~ A f t e r ~} \mathrm{Cl}_{2} \mathrm{MDP}$ or saline i.p. injection, mice were subjected to $\mathrm{ICH}$, and neurological outcomes were evaluated by focal deficit and sticky tape removal time at 1 and 3 days after ICH ( $n=5$ for saline and $\mathrm{Cl}_{2} \mathrm{MDP}$ groups in ART; $n=4$ for saline group, $n=3$ for $\mathrm{Cl}_{2} \mathrm{MDP}$ group in focal deficit, $\left.{ }^{*} p<0.05\right)$. Data are expressed as mean \pm SEM

[17]. In collagenase-induced ICH, M2 phenotype macrophages expressing Arginase-1, Ym1 and CD206 were obtained at delayed time points ( 3 and $7 \mathrm{dpi}$ ). Thus far, it is not clear why macrophages are activated differently depending on the stroke model. Although purely speculative, it is presumed that the microenvironment of the ischemic-injured brain might be distinct from that of the hemorrhagic brain. In this regard, it is of note that ischemic neurons are able to prime microglia polarization to M1 phenotype [17].

In this study, we did not elucidate how M2 macrophages exert their neuroprotective effects in $\mathrm{ICH}$. It is well-known that M2 polarized macrophages contribute to wound-healing by producing various wound-repair genes [29]. It has been reported that brain-infiltrating macrophages contribute to damaged neurovascular unit 

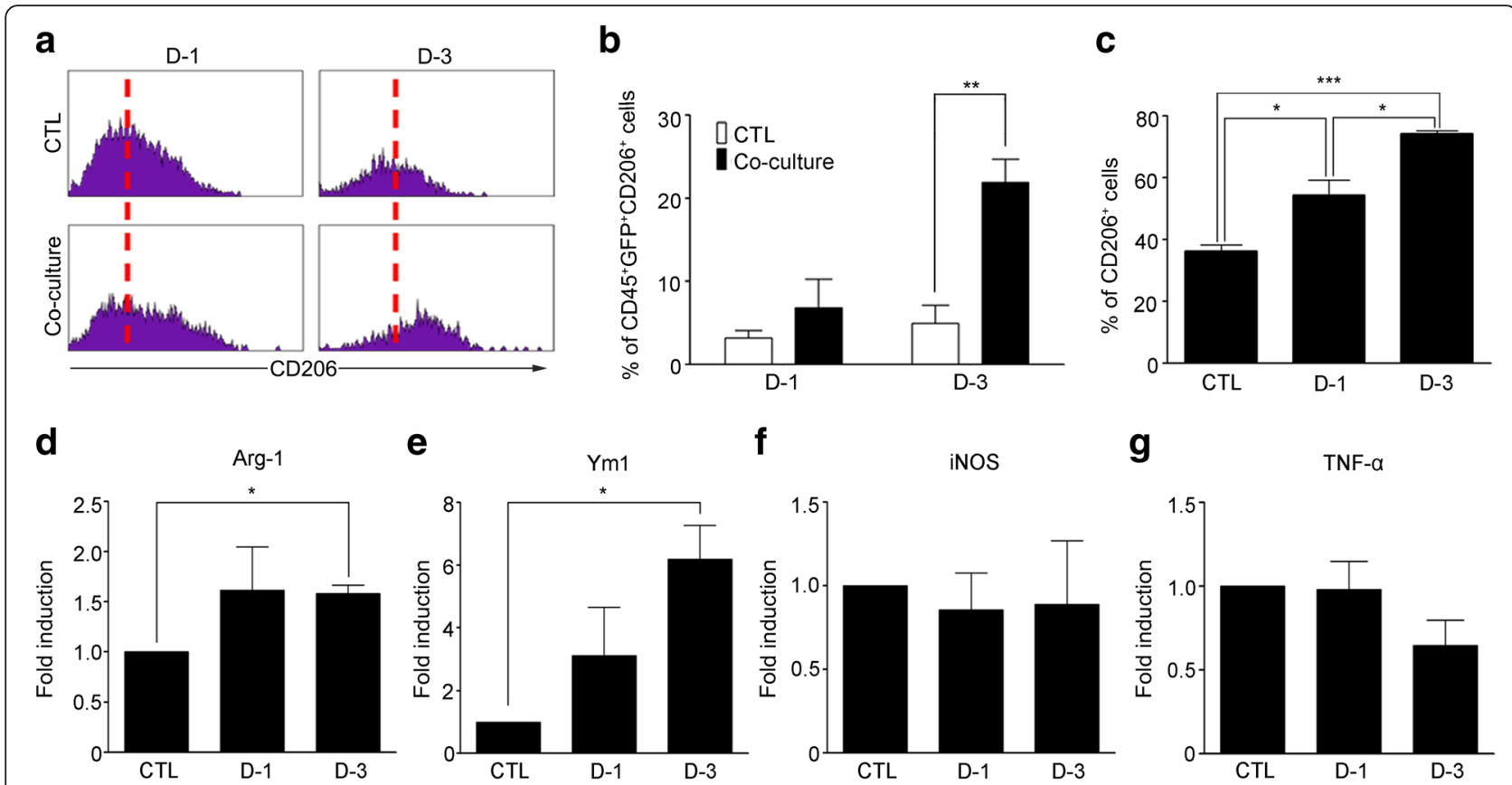

Fig. 4 Soluble factors from mouse brain glial cells induce M2 polarization of BMDM. a BMDM from $C \times 3 \mathrm{Cr}^{+/ G F P}$ mice were cultured with or without primary glial cells from C57BL/6 mice in a 1:1 ratio for 1 or 3 days. Then the expression of CD206 (a M2 marker) was analyzed using flow cytometry. Representative histograms of CD206 expression in GFP ${ }^{+} / C D 45^{+}$populations are shown. $\mathbf{b}$ The percentages of CD206 ${ }^{+}$populations in BMDM were calculated. Mean \pm SEM of 3 independent experiments are shown $\left({ }^{*} p<0.05\right)$. c BMDM were incubated in primary glia-conditioned media for 1 and 3 days. Then, the cells were harvested and stained with CD45-PE, CD11b-FITC, and CD206-APC for flow cytometry analysis. The percentages of $\mathrm{CD}_{20} 6^{+}$cells in $\mathrm{CD} 45^{+} / \mathrm{CD} 11 \mathrm{~b}^{+}$populations are analyzed. Mean $\pm \mathrm{SEM}$ of 3 independent experiments are shown $\left({ }^{*} p<0.05\right.$, $\left.{ }^{* * *} p<0.001\right)$. $\mathbf{d}-\mathbf{g}$ Total RNA was isolated from BMDM at 1 or 3 days after glia-conditioned media treatment. Arg-1, Ym1, iNOS, and TNF-a mRNA expressions were measured using real-time RT-PCR. Mean \pm SEM of at least 3 independent experiments are shown ( ${ }^{*} p<0.05$ )

repair through TGF- $\beta 1$ secretion [30]. Therefore, it is possible that brain-infiltrating M2 macrophages reduce infarct damage by contributing to the wound-healing process in the injured brain tissue. In addition, studies have shown that M2 polarized microglia protect neurons by secreting neurotrophic factors such as BDNF or IGF $[31,32]$. In this regard, it is also conceivable that M2 macrophages protect neurons from ICH injury by producing such neurotrophic factors.

In our effort to elucidate mechanisms of macrophage M2 polarization in the $\mathrm{ICH}$ brain, we found that gliaderived soluble factors have a tendency to drive M2 polarization of BMDM. After infiltration into inflamed tissue, the phenotype and activation of macrophages can be dynamically changed by the microenvironment of the recruiting tissue $[33,34]$. In $\mathrm{ICH}$-injured brains, braininfiltrating macrophages most likely encounter a brain microenvironment consisting of glial cells in the perihematoma region $[35,36]$. Because a co-culture system is widely accepted to study the influence of tissue microenvironment on macrophages, we postulated that a mixed glia culture in vitro might recapitulate the brain microenvironment of brain-infiltrating macrophages during $\mathrm{ICH}$, and tested macrophage polarization in coculture with mixed glia. Indeed, our data showed that primary glial cell-conditioned media can induce the macrophage M2 phenotype. These data are in line with a previous document showing that astrocytes can induce M2 activation of BMDM via the upregulation of miR124 [25]. In addition to the glia-derived factors, various neuron-derived factors are implicated in driving the macrophage M2 phenotype, including CX3CL1 and fragmented myelin $[37,38]$. In this regards, we do not exclude the possibility that macrophages are polarized to M2 phenotype due to neuron-derived molecules in the $\mathrm{ICH}$-injured brain. Although we have not clearly demonstrated the mechanism, our data suggest that braininfiltrating macrophages are polarized to the M2 phenotype due to the microenvironment of the ICH-injured brain, which facilitates recovery from $\mathrm{ICH}$ injury.

\section{Conclusions}

In this study, we found that alternatively activated (M2) macrophages accumulate in the ICH-injured brain. These macrophages contribute to recovery from $\mathrm{ICH}$ at a delayed time. Additionally, we showed that soluble factors released from glial cells drive macrophages to the M2 phenotype. Our data suggest that brain-infiltrating macrophages are polarized to the M2 phenotype and facilitate recovery after $\mathrm{ICH}$. 


\section{Abbreviations}

ART: adhesive removal test; BBB: blood brain barrier; BMDM: bone marrow-derived macrophages; CD206: mannose receptor; $\mathrm{Cl}_{2}$ MDP: clodronate liposome; DMEM: Dulbecco's Modified Eagle's medium; dpi: day post-injury; ICH: intracerebral hemorrhage; IL: interleukin; LPS: lipopolysaccharide; M1: classically activated macrophage; M2: alternatively activated macrophage; NEAA: non-essentia amino acids; rhM-CSF: recombinant human macrophage colony stimulating factor; RT: room temperature; TLR2: toll-like receptor 2.

\section{Competing interests}

The authors declare that they have no competing interests.

\section{Authors' contributions}

$\mathrm{HM}$ and $\mathrm{YHJ}$ carried out ICH surgery for injury volume analysis, macrophage depletion, behavioral test, immunohistochemistry, real-time RT-PCR, glia culture, and BMDM culture. I-HC set up the mouse ICH model and analyzed data. SWY designed and analyzed the data. SJL designed and supervised all the experiments and analyzed data. All authors read and approved the final manuscript.

\section{Funding}

This work was supported by the National Research Foundation of Korea (NRF) funded by the Korean government (MSIP) (2013R1A1A2074231 and 2013R1A2A2A01067248) and by DGIST R\&D Program of the Ministry of Science, ICT and Future Planning (15-BD-0402).

\section{Author details}

${ }^{1}$ Department of Neuroscience and Dental Research Institute, School of Dentistry, Seoul National University, 1 Gwanak-ro, Gwanak-gu, Seoul 08826, Republic of Korea. ${ }^{2}$ Department of Convergence Medical Science, College of Oriental Medicine, Kyung Hee University, Seoul 02447, Korea. ${ }^{3}$ Department of Brain Science, Daegu Gyeongbuk Institute of Science and Technology, Daegu 42988, Korea.

Received: 11 April 2016 Accepted: 14 April 2016

Published online: 19 April 2016

\section{References}

1. Qureshi Al, Mendelow AD, Hanley DF. Intracerebral haemorrhage. Lancet. 2009;373:1632-44.

2. Xi G, Keep RF, Hoff JT. Mechanisms of brain injury after intracerebral haemorrhage. Lancet Neurol. 2006;5:53-63.

3. Panickar KS, Norenberg MD. Astrocytes in cerebral ischemic injury: morphological and general considerations. Glia. 2005:50:287-98.

4. Power C, Henry S, Del Bigio MR, Larsen PH, Corbett D, Imai Y, Yong WW, Peeling J. Intracerebral hemorrhage induces macrophage activation and matrix metalloproteinases. Ann Neurol. 2003;53:731-42.

5. Barone FC, Feuerstein GZ. Inflammatory mediators and stroke: new opportunities for novel therapeutics. J Cereb Blood Flow Metab. 1999:19:819-34.

6. Wang J, Tsirka SE. Contribution of extracellular proteolysis and microglia to intracerebral hemorrhage. Neurocrit Care. 2005;3:77-85

7. Wang J. Preclinical and clinical research on inflammation after intracerebral hemorrhage. Prog Neurobiol. 2010;92:463-77.

8. Min H, Hong J, Cho IH, Jang YH, Lee H, Kim D, Yu SW, Lee S, Lee SJ. TLR2induced astrocyte MMP9 activation compromises the blood brain barrier and exacerbates intracerebral hemorrhage in animal models. Mol Brain. 2015;8:23.

9. Joice SL, Mydeen F, Couraud PO, Weksler BB, Romero IA, Fraser PA, Easton AS. Modulation of blood-brain barrier permeability by neutrophils: in vitro and in vivo studies. Brain Res. 2009;1298:13-23.

10. Wang J, Dore S. Inflammation after intracerebral hemorrhage. J Cereb Blood Flow Metab. 2007;27:894-908

11. Perry VH, Nicoll JA, Holmes C. Microglia in neurodegenerative disease. Nat Rev Neurol. 2010;6:193-201.

12. Kigerl KA, Gensel JC, Ankeny DP, Alexander JK, Donnelly DJ, Popovich PG. Identification of two distinct macrophage subsets with divergent effects causing either neurotoxicity or regeneration in the injured mouse spinal cord. J Neurosci. 2009;29:13435-44.
13. Ding AH, Nathan CF, Stuehr DJ. Release of reactive nitrogen intermediates and reactive oxygen intermediates from mouse peritoneal macrophages. Comparison of activating cytokines and evidence for independent production. J Immunol. 1988:141:2407-12.

14. Sica A, Schioppa T, Mantovani A, Allavena P. Tumour-associated macrophages are a distinct $M 2$ polarised population promoting tumour progression: potential targets of anti-cancer therapy. Eur J Cancer. 2006;42:717-27.

15. Kroner A, Greenhalgh AD, Zarruk JG, Passos Dos Santos R, Gaestel M, David S. TNF and increased intracellular iron alter macrophage polarization to a detrimental M1 phenotype in the injured spinal cord. Neuron. 2014:83:1098-116

16. Stirling DP, Cummins K, Mishra M, Teo W, Yong WW, Stys P. Toll-like receptor 2-mediated alternative activation of microglia is protective after spinal cord injury. Brain. 2014;137:707-23.

17. Hu X, Li P, Guo Y, Wang H, Leak RK, Chen S, Gao Y, Chen J. Microglia/ macrophage polarization dynamics reveal novel mechanism of injury expansion after focal cerebral ischemia. Stroke. 2012;43:3063-70.

18. Bouet V, Boulouard M, Toutain J, Divoux D, Bernaudin M, Schumann-Bard P, Freret T. The adhesive removal test: a sensitive method to assess sensorimotor deficits in mice. Nat Protoc. 2009:4:1560-4.

19. Clark W, Gunion-Rinker L, Lessov N, Hazel K. Citicoline treatment for experimental intracerebral hemorrhage in mice. Stroke. 1998:29:2136-40

20. Cho IH, Hong J, Suh EC, Kim JH, Lee H, Lee JE, Lee S, Kim CH, Kim DW, Jo EK, Lee KE, Karin M, Lee SJ. Role of microglial IKKbeta in kainic acid-induced hippocampal neuronal cell death. Brain. 2008;131:3019-33.

21. Livak KJ, Schmittgen TD. Analysis of relative gene expression data using real-time quantitative PCR and the 2(-Delta Delta C(T)) Method. Methods. 2001:25:402-8.

22. Lee SJ, Zhou T, Choi C, Wang Z, Benveniste EN. Differential regulation and function of Fas expression on glial cells. J Immunol. 2000;164:1277-85.

23. Marim FM, Silveira TN, Lima Jr DS, Zamboni DS. A method for generation of bone marrow-derived macrophages from cryopreserved mouse bone marrow cells. PLoS One. 2010;5:e15263.

24. Bedard A, Tremblay P, Chernomoretz A, Vallieres L. Identification of genes preferentially expressed by microglia and upregulated during cuprizoneinduced inflammation. Glia. 2007;55:777-89.

25. Ponomarev ED, Veremeyko T, Barteneva N, Krichevsky AM, Weiner HL. MicroRNA-124 promotes microglia quiescence and suppresses EAE by deactivating macrophages via the C/EBP-alpha-PU.1 pathway. Nat Med. 2011:17:64-70.

26. Hammond MD, Taylor RA, Mullen MT, Ai Y, Aguila HL, Mack M, Kasner SE, McCullough LD, Sansing LH. CCR2+ Ly6C(hi) inflammatory monocyte recruitment exacerbates acute disability following intracerebral hemorrhage. J Neurosci. 2014:34:3901-9.

27. Sheel M, Engwerda CR. The diverse roles of monocytes in inflammation caused by protozoan parasitic diseases. Trends Parasitol. 2012;28:408-16.

28. Hammond MD, Ai Y, Sansing LH. Gr1+ Macrophages and Dendritic Cells Dominate the Inflammatory Infiltrate 12 Hours After Experimental Intracerebral Hemorrhage. Transl Stroke Res. 2012;3:s125-31.

29. Forbes SJ, Rosenthal N. Preparing the ground for tissue regeneration: from mechanism to therapy. Nat Med. 2014;20:857-69.

30. Gliem M, Mausberg AK, Lee Jl, Simiantonakis I, van Rooijen N, Hartung HP Jander S. Macrophages prevent hemorrhagic infarct transformation in murine stroke models. Ann Neurol. 2012;71:743-52.

31. Cunningham CL, Martinez-Cerdeno V, Noctor SC. Microglia regulate the number of neural precursor cells in the developing cerebral cortex. J Neurosci. 2013:33:4216-33.

32. Suh HS, Zhao ML, Derico L, Choi N, Lee SC. Insulin-like growth factor 1 and 2 (IGF1, IGF2) expression in human microglia: differential regulation by inflammatory mediators. J Neuroinflammation. 2013;10:37.

33. Davis MJ, Tsang TM, Qiu Y, Dayrit JK, Freij JB, Huffnagle GB, Olszewski MA. Macrophage M1/M2 polarization dynamically adapts to changes in cytokine microenvironments in Cryptococcus neoformans infection. MBio. 2013;4:e00264-00213.

34. Gocheva V, Wang HW, Gadea BB, Shree T, Hunter KE, Garfall AL, Berman $T$, Joyce JA. IL-4 induces cathepsin protease activity in tumor-associated macrophages to promote cancer growth and invasion. Genes Dev. 2010;24:241-55. 
35. Haan N, Zhu B, Wang J, Wei X, Song B. Crosstalk between macrophages and astrocytes affects proliferation, reactive phenotype and inflammatory response, suggesting a role during reactive gliosis following spinal cord injury. J Neuroinflammation. 2015;12:109.

36. Gadani SP, Walsh JT, Smirnov I, Zheng J, Kipnis J. The glia-derived alarmin IL-33 orchestrates the immune response and promotes recovery following CNS injury. Neuron. 2015;85:703-9.

37. Boven LA, Van Meurs M, Van Zwam M, Wierenga-Wolf A, Hintzen RQ, Boot RG, Aerts JM, Amor S, Nieuwenhuis EE, Laman JD. Myelin-laden macrophages are anti-inflammatory, consistent with foam cells in multiple sclerosis. Brain. 2006;129:517-26.

38. Wang Y, Fu Y, Xue S, Ai A, Chen H, Lyu Q, Kuang Y. The M2 polarization of macrophage induced by fractalkine in the endometriotic milieu enhances invasiveness of endometrial stromal cells. Int J Clin Exp Pathol. 2014;7:194-203.

Submit your next manuscript to BioMed Central and we will help you at every step:

- We accept pre-submission inquiries

- Our selector tool helps you to find the most relevant journal

- We provide round the clock customer support

- Convenient online submission

- Thorough peer review

- Inclusion in PubMed and all major indexing services

- Maximum visibility for your research

Submit your manuscript at www.biomedcentral.com/submit
C) Biomed Central 